\title{
A NOTE ON THE PAPER GENERALIZED RAYLEIGH PROCESSES
}

Quarterly of Applied Mathematics, XVI, 137 - 145 (1958)

By K. S. MILLER, R. I. BERNSTEIN and L. E. BLUMENSON

In our paper we obtain an explicit representation for the third order density function of a Rayleigh distribution with zero mean. It has come to our attention that a symbolic representation for the $n$th order density function of a Rayleigh distribution with zero mean has appeared in

A. S. Krishnamoorthy and M. Parthasarathy, A multi-variate Gamma type distribution, Annals of Mathematical Statistics, 22, 549-557, (1951).

We would also like to take this opportunity to include the following additional references:

P. B. Patnaik, The non-central $\chi^{2}$ and $F$ distributions and their applications, Biometrika, 36, 202-232, (1949).

W. F. Kibble, A two-variate Gamma type distribution, Sankhya, 5, 137-150, (1941). M. Siotani, Notes on multivariate confidence bounds, Annals of the Institute of Statistical Mathematics, 10, 183-208, (1959).

P. R. Krishnaiah and M. R. Rao, Remarks on a multivariate Gamma distribution, American Mathematical Monthly, 68, No. 4, 342-346, (1961). 